\title{
Aprovechamiento de cenizas volantes y sedimentables provenientes de la valorización energética de cáscaras de maní, como materia prima de la industria cerámica.
}

\section{Use of fly and bottom ashes from the energetic valorisation of peanut shells, as raw material for the ceramic industry.}

Presentación: 6/10/2020

\section{Doctorando:}

\section{Laura Bárbaro}

Grupo de Estudios Ambientales, Facultad Regional San Nicolás, Universidad Tecnológica Nacional, San Nicolás, Argentina. lbarbaro@frsn.utn.edu.ar

\section{Director/a:}

\section{Nancy Quaranta}

\section{Co-director/a:}

\section{Gisela Pelozo}

\section{Resumen}

Las industrias de la región productora de maní, luego de su recolección primaria y posterior procesamiento, acumulan como residuo de este proceso un gran volumen de cáscaras de maní, que han comenzado a ser quemadas con el fin de aprovecharlas para producción de energía. Los residuos inorgánicos que se originan en dicho proceso biomasa-energía, son cenizas volantes o finas y cenizas sedimentables o gruesas, y no tienen al momento un aprovechamiento eficaz, por lo que se propone establecer la factibilidad de su reutilización como materia prima para la fabricación de materiales cerámicos. Se propone, además de la necesidad de resolver la actual disposición final de estos residuos secundarios inorgánicos desde una visión netamente ambiental, valorizar dichos residuos como materia prima de la industria de la construcción, e involucrar en el desarrollo de este trabajo el concepto de economía circular y todos los beneficios asociados a su aplicación a través de herramientas tales como análisis de ciclo de vida (ACV) y huella de carbono, entre otras.

Palabras clave: Materiales cerámicos, Economía circular, Cenizas de maní, Biomasa

\begin{abstract}
The industries of the peanut-producing region, after their primary harvesting and subsequent processing, accumulate as a waste from this process a large volume of peanut shells, which have begun to be burned in order to take advantage of them for energy production. The inorganic residues originated in this biomass-energy process, are fly or fine ash and bottom or coarse ash, and do not have an effective use at the moment, so it is proposed to establish the feasibility of its reuse as raw material for manufacturing ceramic materials. In addition to the need to resolve the current final disposal of these inorganic secondary waste from a purely environmental perspective, it is proposed to value these wastes as a raw material for the construction industry, and to involve the
\end{abstract}


concept of circular economy in the development of this work, and all the benefits associated with its application through tools such as life cycle assessment (LCA) and carbon footprint among others.

Keywords: Ceramic materials, Circular economy, Peanut ashes, Biomass

\section{Introducción}

El uso de combustibles fósiles ha sido el método convencional de producción de energía durante muchos años, sin embargo, durante las últimas décadas, la escasez de los recursos energéticos fósiles, el impacto ambiental negativo derivado de su uso y su variabilidad de precios han alentado la búsqueda de fuentes alternativas de energía de acuerdo con sistemas renovables, sostenibles, eficientes y rentables. Entre los recursos renovables, la biomasa se caracteriza por su gran potencial energético, que puede utilizarse para producir energía térmica, eléctrica y biocarburantes. Se considera que el proceso de producción de energía por combustión de biomasa tiene importantes ventajas medioambientales, sin embargo, también tiene la desventaja de generar grandes cantidades de cenizas. En consecuencia, la producción de ceniza se convierte en un problema medioambiental si no se gestiona adecuadamente.

El ciclo de vida de un producto normalmente comienza cuando los recursos naturales son extraídos (por ejemplo, minería) o producidos (por ejemplo, producción agropecuaria); luego esta materia prima se transporta y procesa para ser usada o consumida, y finalmente termina con la disposición final del residuo, o su reciclado o reúso. En cada fase del ciclo de vida de un producto, existen emisiones y consumo de recursos. El Análisis de Ciclo de Vida (ACV) es una técnica de relevamiento y análisis de datos (emisiones y consumos) de las etapas necesarias para que el producto sea obtenido, utilizado y finalmente descartado. Esta técnica evalúa los aspectos ambientales e impactos potenciales de un producto, un proceso o un servicio, a lo largo de un periodo de tiempo (Fernandez \& Giayetto, 2017).

Se prevé que para el año 2050, la población mundial supere los 9 mil millones de personas, de las cuales el 66 por ciento vivan en ciudades. Acompañando este impresionante ritmo de urbanización, habrá una compleja red de desafíos relacionados con el consumo, la contaminación y el estrés hídrico y energético (United Nations, Department of Economic and Social Affairs, Population Division, 2015). Es así como la idea de una transición a una economía circular no solo equivale a ajustes destinados a reducir los impactos negativos de la economía lineal, sino por el contrario, representa un cambio sistémico que construye resiliencia a largo plazo, genera oportunidades comerciales y económicas, y proporciona beneficios ambientales y sociales. La economía circular se presenta como una alternativa al actual modelo de producción y consumo, con el potencial de resolver retos medioambientales, al mismo tiempo que abre oportunidades de negocio y crecimiento económico. Se propone como la alternativa lógica y viable, que corrige los principales problemas de la linealidad y pretende conseguir que los productos, componentes y recursos en general mantengan su utilidad y valor en todo momento, o lo que es lo mismo, conduce al concepto de residuo cero (Morocho, 2018).

En este trabajo, este análisis es propuesto ante una necesidad de solución a problemas asociados a un tipo de residuo secundario de biomasa muy abundante, principalmente en la región central de nuestro país, en especial la provincia de Córdoba, que en este momento se acopia y se envía a disposición final. Las industrias de la región productora de maní, luego de su producción primaria y posterior procesamiento, acumulan como residuo de este proceso un gran volumen de cáscaras de maní, que ya han comenzado a ser quemadas con el fin de aprovecharlas para producción de energía. Los residuos inorgánicos que se originan en dicho proceso biomasa-energía, son cenizas volantes o finas y cenizas sedimentables o gruesas, y no tienen al momento un aprovechamiento eficaz.

Se detecta la necesidad de resolver la actual situación de disposición de estos residuos secundarios inorgánicos desde una visión principalmente ambiental. Se propone establecer la factibilidad de su reutilización como materia prima para la fabricación de 
materiales cerámicos, involucrando en el desarrollo de este trabajo el concepto de economía circular y todos los beneficios asociados a su aplicación.

Es importante destacar que uno de los objetivos de este trabajo es interactuar con las empresas que producen estos descartes secundarios de procesos biomasa-energía, que utilizan diferentes biomasas, con el fin de generar conciencia sobre los problemas que provocan las disposiciones de los mismos, tratando de cambiar la mirada netamente económica que por lo general se detecta en estos ámbitos. En ese sentido es uno de los resultados esperados del trabajo el despertar el interés de los profesionales encargados del sector medioambiental, incentivando y proponiendo el análisis de estos procesos desde los conceptos de valorización de residuos, balances ambientales, huellas de carbono y economía circular.

\section{Desarrollo}

Este trabajo se ha planteado luego de la búsqueda de información que condujo a establecer el estado actual de desarrollo de procesos biomasa-energía en el mundo en general, y en Argentina en particular. En ese sentido el Comité de Biomasa de la Cámara Argentina de Energías Renovables (CADER), indica que nuestro país posee aproximadamente 80 plantas de biomasa, de las cuales 20 son grandes instalaciones. Estas plantas son básicamente de tratamiento de residuos sólidos urbanos o componentes orgánicos, así como también mini-biodigestores o minilagunas cubiertas para consumo domiciliario (ENEL Argentina, 2018).

Resultan muy pocos los casos de empresas que utilizan sus propios residuos de proceso como combustible para producir energía, y en la mayoría de ellos, dicha energía es aprovechada para consumo interno de la propia planta. Uno de los casos emblemáticos detectados es el de la empresa PRODEMAN S.A., que no solamente produce energía para su propio consumo, sino que vuelca el sobrante a la red nacional de distribución, e inclusive ha comenzado a recibir descartes de cáscaras de maní de otras empresas. El contacto realizado con los ingenieros responsables de este proceso, ha dado origen al plan de trabajo planteado en esta investigación.

La Figura 1 muestra un esquema simple de las etapas propuestas, que consiste en primer lugar en el análisis del proceso productivo primario y sus descartes (cáscaras de maní), luego el estudio del proceso biomasa-energía y la obtención de los materiales residuales correspondientes, constituidos básicamente por cenizas volantes y sedimentables, que se recogen separadamente en la planta. Continuando con el desarrollo se plantea la etapa de caracterización exhaustiva de estas cenizas, para finalmente realizar la valorización de las mismas, como agregados en mezclas arcillosas para la producción de piezas cerámicas.

Para dar contexto al análisis del proceso productivo de la mencionada empresa, puede describirse la misma como una empresa manisera familiar, que desde 1984 produce, selecciona, acopia, procesa y exporta alimentos, cumpliendo con los estándares de inocuidad y calidad, y bajo las especificaciones y requerimientos exigentes de los mercados internacionales, respetando el medio ambiente y el desarrollo personal de los recursos humanos.

En su planta desarrollan actividades un gran número de personas, que llevan adelante los procesos productivos y de comercialización del maní. La planta de producción está ubicada en un predio de 24 hectáreas con un total de $90.000 \mathrm{~m}^{2}$ cubiertos, y está conformada por diversas plantas de productos tales como maní tipo confitería, maní blancheado, y otros productos más elaborados. Posee además galpones de almacenamiento de insumos, galpones refrigerados para el almacenamiento de 40.000 toneladas de productos, celdas de acopio de materia prima con capacidad para 110.000 toneladas, un taller montado para el mantenimiento de las plantas, y laboratorios de control de calidad, microbiología, cromatografía gaseosa y análisis fisicoquímicos (PRODEMAN S.A., 2019). 


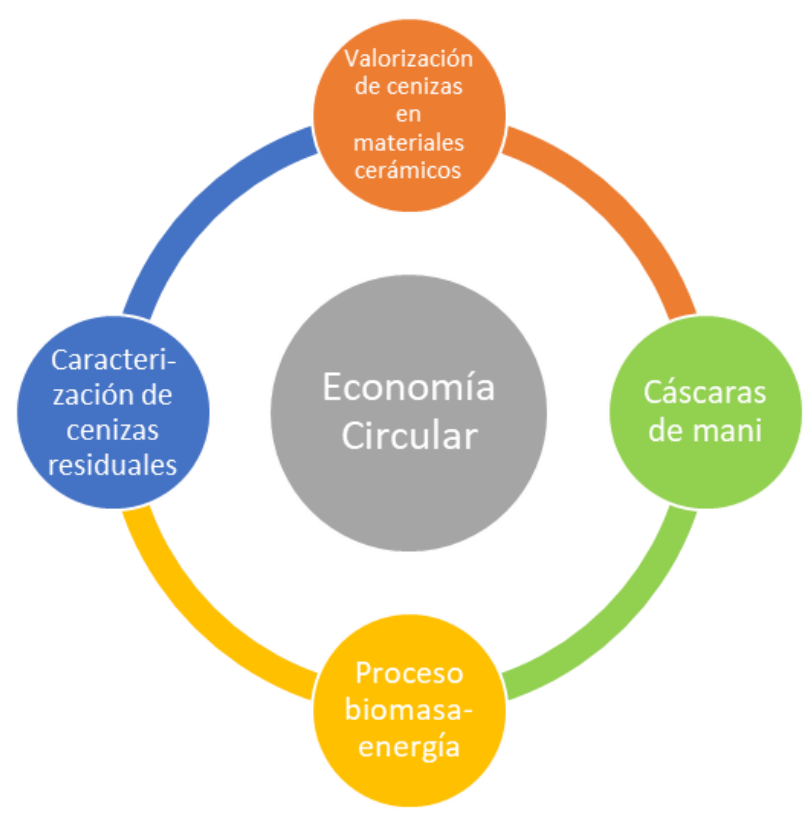

Figura 1. Esquema de las etapas de trabajo

En 2017, esta empresa puso en marcha una planta de generación de energía eléctrica a base de cáscaras de maní (Figura 2). La usina cuenta con una turbina de vapor de 10 megavatios de potencia, con capacidad para generar 78.840 MW/hora. La empresa usa el $10 \%$ de la energía para su funcionamiento, el 25\% para el proceso de industrialización del maní y el $65 \%$ restante se incorpora a la red nacional de electricidad. La energía obtenida por la transformación de esta biomasa se incorpora al sistema interconectado nacional, abasteciendo a 18.000 hogares por año. Para generar energía necesita aproximadamente 50.000 toneladas de cáscara de maní al año. Para ello, las cáscaras de maní se acopian en celdas y desde allí se trasladan a una caldera donde se quema y se transforma en energía potencial de vapor de agua, que se traslada a la turbina de vapor donde se convierte en energía mecánica de rotación, que acoplada al generador se termina transformando en energía eléctrica (PRODEMAN S.A., 2019).

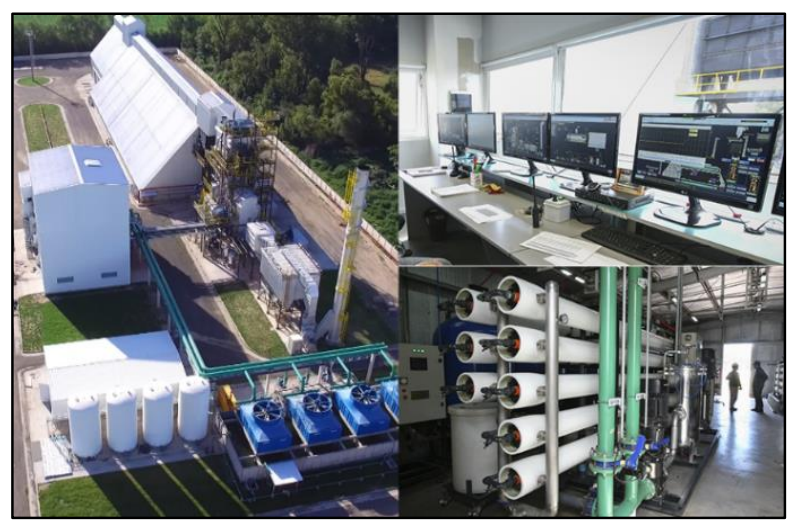

Figura 2. Planta de generación de energía eléctrica con biomasa

Si bien todavía falta un marco legislativo y de financiamiento para el sector, los proyectos para la producción de energía a partir de biomasa residual siguen en crecimiento y permitirán una mayor generación de energía limpia en nuestro país. 
En este contexto, uno de los objetivos de este trabajo es caracterizar las cenizas residuales provenientes de la combustión de cáscaras de maní y estudiar su factibilidad de incorporación en mezclas arcillosas para la producción de materiales cerámicos.

Las cenizas volantes (CV) y sedimentables (CS) utilizadas en el estudio propuesto provienen de la mencionada empresa, en particular de la usina que se ha descripto. Estos materiales residuales se caracterizarán mediante diferentes técnicas entre las cuales se encuentran microscopía óptica (OM) y electrónica de barrido (SEM), análisis químico semicuantitativo por espectrometría de dispersión de energía de rayos X (EDS), pérdida de peso por calcinación (LOI), análisis de difracción de rayos X (XRD), análisis térmico diferencial y termogravimétrico (DTA-TGA). Además, con el fin de determinar la factibilidad de incorporación de las cenizas en mezclas arcillosas se estudiarán las temperaturas teóricas de sinterización de dichas mezclas.

\section{Resultados}

El análisis químico por EDS de las cenizas se presenta en la Tabla 1. Puede observarse que ambas están compuestas además de $\mathrm{C}$ y O, por importantes porcentajes de Si y Ca. En menor proporción se observa Fe, $\mathrm{Na}, \mathrm{Mg}, \mathrm{Al}, \mathrm{P}, \mathrm{S}$ y K.

Los diagramas de difracción de rayos $\mathrm{X}$ de estas cenizas muestran la presencia de diversas fases cristalinas: cuarzo y silicato de calcio $\left(\mathrm{Ca}_{2} \mathrm{SiO}_{4}\right)$ en las $\mathrm{CV}$, y cuarzo, sílice, calcita y silicato de potasio $\left(\mathrm{K}_{6} \mathrm{Si}_{3} \mathrm{O}_{9}\right)$ en las CS.

\begin{tabular}{|c|c|c|c|c|c|c|c|c|c|c|c|}
\hline & $\mathrm{C}$ & $\mathrm{O}$ & $\mathrm{Fe}$ & $\mathrm{Na}$ & $\mathrm{Mg}$ & $\mathrm{Al}$ & $\mathrm{Si}$ & $\mathrm{P}$ & $\mathrm{S}$ & $\mathrm{K}$ & $\mathrm{Ca}$ \\
\hline $\mathrm{CV}$ & 10.9 & 31.6 & 5.1 & 1.1 & 4.4 & 5.2 & 18.5 & 1.4 & 1.1 & 9.2 & 11.5 \\
\hline $\mathrm{CS}$ & 3.9 & 32.6 & 9.9 & 2.6 & 10.5 & 2.5 & 13.5 & 2.7 & 0.5 & 4.9 & 16.4 \\
\hline
\end{tabular}

Tabla 1. Análisis químico por EDS de las cenizas.
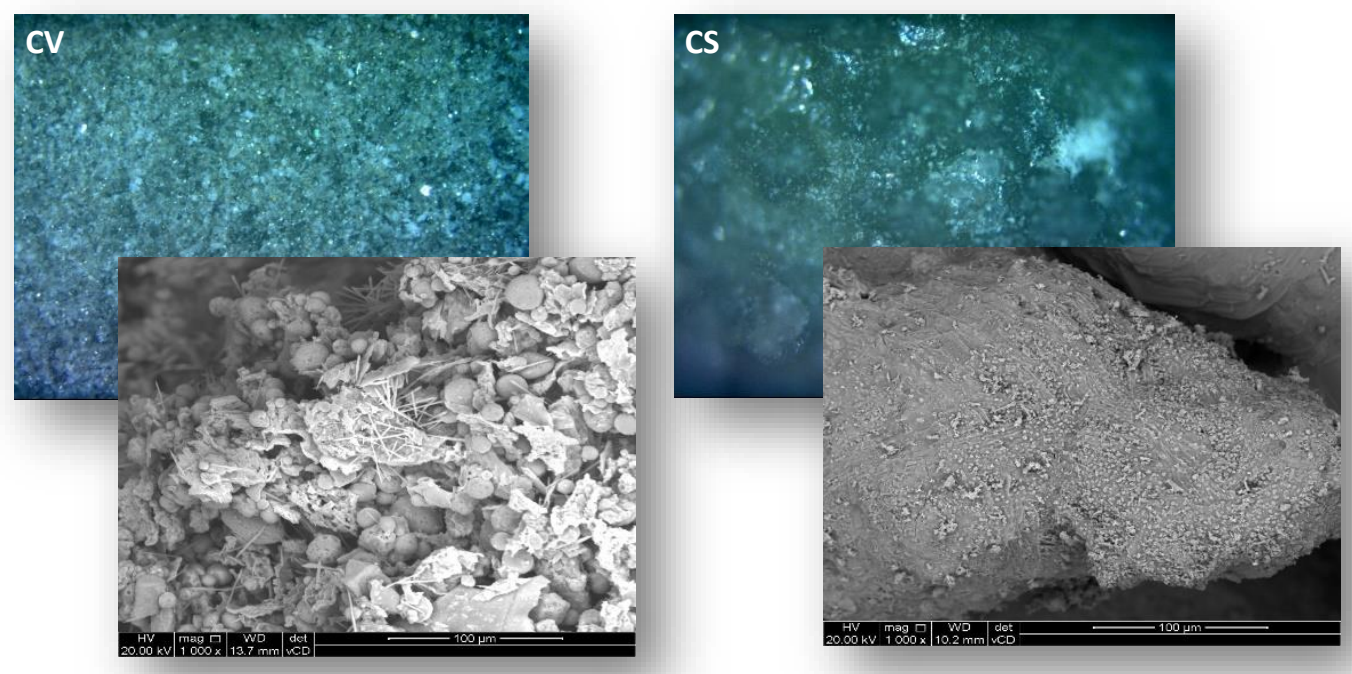

Figura 3. Micrografías OM (50X) y SEM de cenizas volantes (CV) y sedimentables (CS).

Las imágenes de microscopía OM y SEM muestran que las cenizas volantes están conformadas por partículas de forma esférica y acicular de tamaño homogéneo, mientras que las cenizas sedimentables son un conglomerado de partículas más pequeñas, como puede observarse en la Figura 3. 
El análisis térmico diferencial y termogravimétrico de ambas cenizas presenta una reacción exotérmica alrededor de $200^{\circ} \mathrm{C}$, debido a la evaporación del agua adsorbida en las muestras (Figura 4). La muestra CV presenta además un pico exotérmico a $400^{\circ} \mathrm{C}$ asociado a la combustión de la fracción no quemada durante el proceso. La muestra CS no presenta esta reacción ya que el contenido de $\mathrm{C}$ es mucho menor (3,9\%), en concordancia con los valores de pérdida de peso por calcinación (LOI), que para el caso de las CS fue de $0 \%$ y para las CV $8,5 \%$.
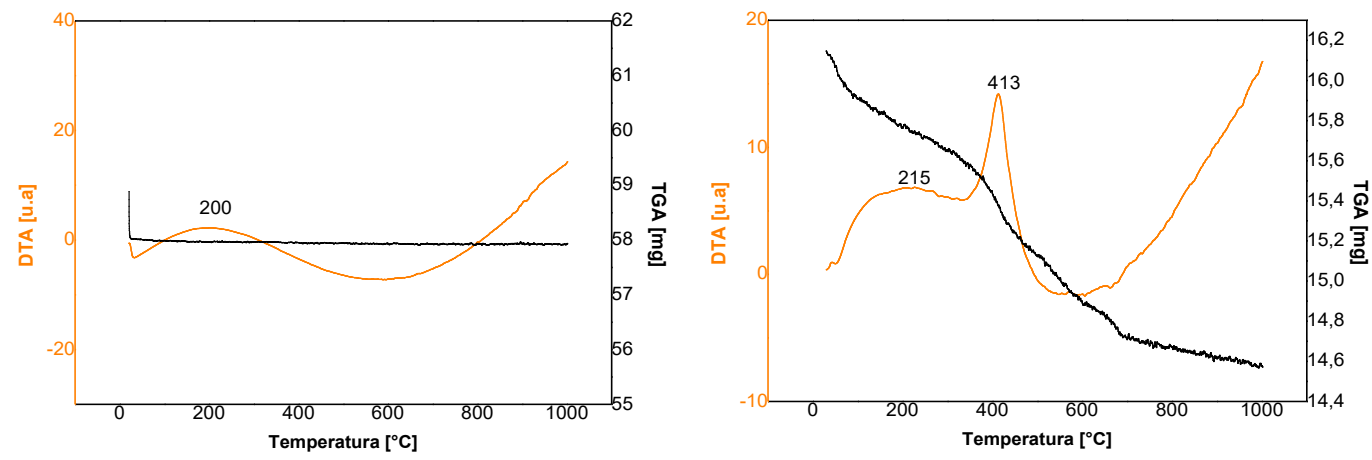

Figura 4. DTA-TGA de las cenizas CV (derecha) y CS (izquierda).

Con el propósito de determinar la temperatura de sinterización teórica de mezclas cenizas-arcilla, se tomaron los óxidos mayoritarios de una arcilla comercial y los de las cenizas y se ubicaron las mezclas con $10 \%$ de cenizas en un diagrama de equilibrio ternario $\mathrm{SiO}_{2}-\mathrm{Al}_{2} \mathrm{O}_{3}-\mathrm{FeO} \cdot \mathrm{Fe}_{2} \mathrm{O}_{3}$. La temperatura de sinterización resultante fue de aproximadamente $1000^{\circ} \mathrm{C}$.

\section{Conclusiones}

En este trabajo de Tesis, se ha comenzado con el análisis de los procesos productivos de la industria manisera, y de su industria satélite de producción de energía a partir de la combustión de las cáscaras de maní. Se han obtenido los residuos del proceso biomasa-energía, constituidos por cenizas volantes y sedimentables, y se ha comenzado con la caracterización de estos materiales.

Los resultados de los ensayos de caracterización de las cenizas realizados hasta el momento permiten continuar en el camino de determinar la factibilidad de su valorización como materia prima de la industria de la construcción.

\section{Referencias}

ENEL Argentina, C. 1. (2018). https://www.enel.com.ar/es/Historias/a201802-biomasa-en-argentina-con-80-plantas.html. Consulta realizada en noviembre de 2019.

Fernandez, E. M., \& Giayetto, O. (2017). El cultivo de maní en Córdoba. Ed. Universidad Nacional de Rio Cuarto. https://www.produccionvegetalunrc.org/docs/ECMC_2.pdf

Morocho, F. R. (2018). La Economía Circular Como Factor De Desarrollo Sustentable Del Sector Productivo. Ecuador: INNOVA Research Journal 3 (12) 78-98. https://doi.org/10.33890/innova.v3.n12.2018.786

PRODEMAN S.A. https://prodeman.com. Consulta realizada en noviembre 2019.

United Nations, Department of Economic and Social Affairs, Population Division (2015). World Urbanization Prospects: The 2014 Revision, (ST/ESA/SER.A/366). https://population.un.org/wup/Publications/Files/WUP2014-Report.pdf 